\title{
XXII. The bakerian lecture. On the relations of electrical and chemical changes
}

\author{
Sir Humphry Davy Bart. Pres. R.S.
}

To cite this article: Sir Humphry Davy Bart. Pres. R.S. (1827) XXII. The bakerian lecture. On the relations of electrical and chemical changes, Philosophical Magazine Series 2, 1:2, 94-104, DOI: $10.1080 / 14786442708674234$

To link to this article: http://dx.doi.org/10.1080/14786442708674234

里 Published online: 10 Jul 2009.

Submit your article to this journal $₫$

Џ Article views: 2

Q View related articles $₫$ 
value of the quantity sought was known, it became a point of great importance to ascertain, by varying the circumstances of the experiment, whether that quantity always retained the same value independently of the different states of the atmosphere; and all the trials that have been made favour the conclusion that it is nearly constant. But the constancy of the factor is now proved $a$ priori by the theory here laid down, and is no longer merely an induction from experiments. Taking $\frac{\alpha}{\beta}=\frac{3}{8}$, we are entitled to enunciate the following proposition, which solves the proposed problem:

The heat extricated from air when it undergoes a given condensation, is equal to $\frac{3}{8}$ of the diminution of temperature required to produce the same condensation, the pressure being constant.

Air, under a constant pressure, diminishes $\frac{1}{4} 0$ th of its volume for every degree of depression on Fahrenheit's scale; and therefore one degree of heat will be extricated from air when it undergoes a condensation equal to $\frac{1}{480} \times \frac{3}{8}=\frac{1}{180^{\circ}}$ If a mass of air were suddenly reduced to half its bulk, the heat evolved would be $\frac{1}{2} \div \frac{1}{1} \frac{1}{80}=90^{\circ}$.

Having now solved the proposed problem, I shall reserve what further is important on this subject to a future occasion.

Jan. 8, 1827 .

J. Ivory.

XXII. The Bakerian Lecture. On the Relations of Electrical and Chemical Changes. By Sir Humphry Davy, Bart. Pres. R.S.

[Continued from p. 38.]

IV. On the electrical and chemical effects exhibited by combinations containing single metals and one fuid.

KNOW of no class of phænomena more calculated to give just views of the nature of electro-chemical action than those presented by single metals and fluids; and as their results are, with one or two exceptions, entirely new, I shall describe them with some degree of minuteness. - When two pieces of the same polished copper, connected with the platinum wires of the multiplier, were introduced at the same time into the same solution of hydro-sulphuret of potassa, there was no action; but if they were introduced in succession, there was a distinct and often, if the interval of time was considerable, a violent electrical effect-the piece of metal first plunged in being negative, and the other positive.

This result depends upon the circumstance of the production 
tion of a new combination, which is negative with respect to the metal; for after the formation of the sulphuret of copper, the plate of copper that has been first plunged into the solution exhibits the same negative state with respect to polished copper, whether introduced into saline solutions, or alkaline or acid menstrua. The electrical effect therefore does not depend on so simple a condition as would at first appear, and it may be in fact referred to the combinations containing two metallic substances and one fluid.

The gray sulphuret of copper is negative, in solutions of hydro-sulphuret, to clean copper, and the superficial coating has apparently similar electrical powers to this substance.

Copper, in the state of protoxide, is negative, not only with respect to metallic copper, but likewise with respect to the sulphuret; a circumstance which explains many singular and apparently anomalous circumstances with respect to the action of hydro-sulphuret on copper. I have often found the order which I have mentioned, of metallic copper being positive with respect to copper that had been a few seconds in solution of hydro-sulphuret, reversed in a singular and capricious way; but on investigating the cause, I found that the copper was tarnished; and on heating any kind of polished copper strongly, so as to produce a thin coating of oxide any where on its surface, it became strongly negative to copper plunged in solution of hydro-sulphuret: the same effect was produced by the action of acids.

There are some singular circumstances connected with the violent and intense chemical action of copper on solutions of hydro-sulphurets, which are worthy of being described. When a piece of copper connected with the multiplier has been for a minute in strong solution of hydro-sulphuret of potassa, on introducing a piece of polished copper connected with the other wire, there is often a violent and momentary negative charge communicated to it, which sends the needle through a whole revolution: it then oscillates, and almost immediately returns, and takes the direction which indicates that the piece first plunged in is negative. This effect continues for some minutes, then becomes weaker; at last the two sides are in equilibrium, and the piece which was first plunged in now becomes positive with respect to the other. The first described of these effects seems to depend upon the discharge, by the clean copper, of the negative electricity accumulated by the contact of the plate first plunged in, before the relative states produced by the metallic contact and the regular currents occur; and the second, to the detaching or peeling off of the coat of sulphuret, which has the effect of exposing 
a clean surface, and which effect is probably occasioned by the oxidation of the positive side of the plate.

There are few electrical actions more intense than those produced by the operation of hydro-sulphurets on copper in these different circumstances; so much so, that I have constructed a Voltaic battery which decomposed water, by six combinations, consisting merely of thin slips of copper, of which one half had been exposed to the solution about a minute before the other half: of course, the oxidating surface was on the side of the clean or latest exposed metal.

With lead, and alloys of tin and lead and iron, there are the same phænomena, but much feebler electrical action, the metallic surface which is first introduced being the negative surface; and the principles of this kind of action are precisely the same as those of copper and hydro-sulphurets.

Zinc, platinum, and metals which have no chemical action on solutions of hydro-sulphurets, produce no phænomena of this kind; silver and palladium, which act powerfully with these menstrua, produce very decided effects; but the compounds they form in them being positive with respect to the pure metals, the phænomena are the reverse of those offered by the more oxidable metals; the surface plunged first into the solution is the positive surface, and it retains this relation in alkaline, acid, and saline solutions, presenting peculiarities dependent upon the change of surface, which I shall refer to again hereafter.

The production of electrical currents by single metals and single fluids, though most distinct in the cases $I$ have just named, yet occurs generally whenever new substances which can adhere to the metals are produced in chemical action. Thus in acid solutions of a certain strength pieces of the same zinc, tin, iron, and copper, exhibit similar phænomena; the surface first plunged into the acid being tarnished, or retaining a slight coat of oxide, is negative to the metal plunged in afterwards, and the relation is sustained in saline or alkaline solutious. The same effect is caused by producing a coat of oxide by heat on the surface, or even by applying it artificially. The oxidated surface is negative with respect to the other.

Zinc, which dissolves in a strong solution of potassa, giving off hydrogen copiously, exhibits exactly the same phænomena in this solution; the tarnished metal, or that first introduced, being negative with respective to the other. Tin likewise in solution of potassa, having been introduced long enough to have tarnished, is strongly negative with respect to polished tin.

Even the noble metals obey the same law. Silver, that has 
been tarnished by the action of nitric acid, is negative to polished silver in diluted acid; and gold and platinum, that have been acted on by aqua regia, are negative in that acid to the clean metals.

The intimate connexion displayed in all these cases between the chemical and electrical phænomena, becomes still more remarkable when the nature of the changes taking place in circles of this kind is considered.

Oxygen, which may be considered as negative with respect to all the metals, and sulphux, which is negative with respect to the oxidable metals, by their combinations with metals respectively positive to them, produce compounds negative with regard to those metals. And in the chemical changes, the results are such as must ultimately restore the equilibrium, hydrogen or sulphuretted hydrogen passing to the negative side, and oxygen to the positive side; so that the oxides are revived; and not only is the equilibrium restored, but the poles sometimes changed. Thus tin that has tarnished in acid, remains for some time negative in solution of alkali, but gradually as the oxide upon it is revived by the hydrogen determined to this surface, it loses its negative power; and the other surface, now tarnished by the action of the alkali, gains this power, whilst the opposite surface becomes positive.

\section{Of electrical combinations, consisting of two imperfect, and} one perfect conductor; or two fluids and a metal, or charcoal.

To understand clearly the nature of the action in this kind of electrical combination, it is necessary to consider the nature of imperfect conducting bodies, water, or saline solutions. These bodies may be regarded as having the same relations to electricities of very low intensity, that elastic fluids have to the electricities of glass, sealing-wax, or the common machine. They communicate' the electrical polarities of the metals, but do not appear capable of receiving such polarities, or at least of retaining them; and the electrical equilibrinm, when broken in them, seems to be rapidly restored by a new arrangement or attraction of certain of their elements. For instance, if we introduce the positive and negative poles from a very powerful voltaic battery into the extremities of a basin filled with solution of muriate of lime, and place in the circuit different wires of platinum, every wire will possess a positive and negative pole, and there will be no division of the fluid into two parts, one positive, the other negative; and when the two wires are withdrawn, they alone having been used, the electrical appearances immediately cease; and metallic wires unconnected with the battery made to occupy their places, exhibit no electrical phænomena: and in all experiments of this Nero Series. Vol. 1. No.2. Keb. 1827. 
kind, the well known phænomena of the development of chlorine and oxygen and acid matter at the positive, and hydrogen, alkaline matter, \&c. at the negative pole, takes place.

Acid and alkaline matters, when perfectly dry and nonconducting, become on contact negative and positive; as I have shown is the case with oxalic acid and lime; but this effect is similar to that of glass and silk, and the result is a common electricity of tension. And when acids and alkalies combine, their union being apparently the result of the same attractive powers acting on the particles which would produce their electrical relations as masses, they exhibit no phænomena of electro-motion; and such phænomena, when they occur in combinations in which acids and alkalies unite, always depend upon the contact of the metal with the acid and alkaline matter, change of temperature, evaporation, \&c. and never on the combination of the acid and alkali.

As a different opinion has been lately started, on high authority*, I shall give the proofs of the truth of this my early view, which appear to me of the strictest demonstrative nature.

A solution of nitre, which is a substance neutral to the contact of noble metals, was introduced into a glass cup containing a plate of platinum connected with the multiplier; pure concentrated nitric acid was placed in another cup, in which there was another plate of platinum joined to the other wire of the multiplier, and the connexion was made by a piece of asbestus wetted in a solution of nitre. At the moment of contact, the needle indicated a strong electrical action, negative on the plate plunged in the acid, and which occasioned a permanent deviation of about $60^{\circ}$.

This arrangement was removed from the multiplier, and another substituted for it, in which strong solution of potassa occupied the place of the nitric acid, being in contact with platinum in one cup, and solution of nitre in the other, with the same communications. The deviation was now much weaker, about 10 degrees, and the platinum in the solution of potassa was positive.

The nitric acid and the solution of potassa were now connected in the arrangement by a piece of clean asbestus, moistened in a concentrated solution of nitre; the deviation of the needle was to about $65^{\circ}$. In this instance there was no chemical action of the fluids on each other; for they had no tendency to mix rapidly with the solution of nitre, which being of less specific gravity than either of the other solutions, remained in the asbestus; and there was no effect beyond that of the metallic contact of the platinum with acid and alkali.

* That of M. Becquerel. 
A piece of asbestus, of nearly the same size with the other, but dry, was now substituted for the moist asbestus, so that the acid and alkali combined by capillary attraction producing heat: at first, tlre deviation was rather less than in the former instance; but as soon as the combination was complete, the needle stood exactly at the same point, proving that no electricity was developed by the combination, any more than by the indirect communication of the acid and the alkali.

After trying the effects of the contact of fluid acid upon platinum by the arrangement with solution of nitre, and finding that oxalic acid was the acid among the powerful ones which produced the slightest deviation of the needle, or the smallest negative effect, I employed this acid and solution of potassa, exactly in the same manner as the nitric acid in the experiment just detailed; as the joint action of the acid and alkali on the platinum was only to produce a deviation of 7 or 8 degrees, it might be suspected that any electrical action produced by combination might be more easily manifested; but no such effect occurred; and whether the communication was made by combination through dry asbestus, or through asbestus wetted in a saline solution, the effect was precisely the same.

Again,- - the two surfaces of platinum were placed in contact with strong solutions of nitre, and the communication made between them by solution of potassa and nitric acid; there was no electrical action, though the chemical combination was intense. But when the fluids were mixed, so that a little acid touched one plate of platinum and a little alkali the other, electro-motion immediately began; and in using muriatic acid and solution of ammonia, which, being lighter than the saline solutions, very soon came in contact with the platinum, the effect commenced almost immediately, and continued for some time to increase.

Again,-I placed pieces of paper coloured with litmus and turmeric, and moistened in solutions of nitre, upon two surfaces of platinum connected with the multiplier; they were covered with a stratum of porcelain clay wetted with the same solution, a stratum of clay moistened with muriatic acid was placed above on one plate, and a stratum moistened with solution of ammonia above on the other, so as to make a contact in which there should be action upon a large surface without direct communication with the metals. In several experiments of this kind there was no electro-motion; and whenever it was perceived, it was found that either the acid, or the alkali, or both, had penetrated through the clay, and touched the metals so as to change considerably the colour of 
the papers, which were placed as indications of the correctness of the experiment.

Having brought forward what appear to me decided proofs $\mathrm{n}$ this subject, I shall now proceed to investigate the operation of the metals and fluids in combinations containing two of the latter substances. At first I was surprised to find that platinum acted so powerfully with nitric acid, which undergoes no chemical change by contact with it, and suspecting that it might arise from the presence of minute portions of muriatic acid or muriatic salts, I took great pains to exclude these substances by washing the platinum in distilled water, not touching it with the hands, \&c. but when the conditions were those of perfectly clean and pure platinum and perfectly pure nitric acid, the phænomena were the same. Similar reasonings may be applied to solutions of potassa, soda, \&c. which do not chemically alter platinum by contact, and yet render it positively electrical with respect to platinum in water or saline solutions. It must however be called to mind that the oxygen in nitric acid, and the metals in the alkalies, have attractions of a very decided kind for platinum; and in taking the scale of electro-negative bodies, solutions of chlorine, or nitro-muriatic acid, produce a more powerful electrical effect on platinum than nitric acid, nitric acid than muriatic, and muriatic than sulphuric.

When platinum is brought in contact with an acid, the pole touching the acid is negative, the opposite pole is positive, as I have found by the condensing electrometer; and the reverse is the case when it touches an alkali; so that the circulation of the electricity is from the metal to the alkali, and from the acid to the metal.

Rhodium, iridium, and gold, act in combinations consisting of acid and alkali, on which they have no chemical effect, exactly like platinum; the surface of the metal in the solution of alkali being positive, that in the solution of the acid, negative. With silver and palladium the electricity is greater, particularly if nitric acid is used; and with charcoal and oxidable metals, there is the same general result, the action being in general exalted in proportion as the chemical attractions are stronger, provided there are no interfering circumstances : and in combinations of this kind nitro-muriatic acid is more active than nitric, and the order is after, nitric, nitrous sulphuric, phosphoric, vegetable acids, sulphurous, prussic, sulphuretted hydrogen; and, with the alkalies, potassa, soda, baryta, ammonia, and so on.

It is always to be understood that strong or concentrated solutions of acids and alkalies are employed; for in cases where 
where the quantity of acid or alkaline matter is very small and the chemical action of the metals strong, there is sometimes a different order. Thus zinc and tin tarnish immediately even in a weak solution of potassa, and, so tarnished, they are negative to the same metals in weak solutions of muriatic or sulphuric acid; but in experiments of this kind it is easy to determine the true circumstances by changing the poles; the negative side, when the energies of the alkali and acid are weak, will be determined by the tarnish or coat of oxide formed.

Solutions of sulphurets act in these combinations like alkali, with circumstances depending upon the formation of new compounds, according to the law explained in the last section. In combinations, of which the elements are hydro-sulphuret and acid, the metal in the hydro-sulphuretted solution is positive, and that in the acid negative; but with alkalies and hydro-sulphurets, and zinc and tin, the metal in the solution of alkali is positive, and that in the solution of hydro-sulphuret, negative: with silver and palladium the opposite order occurs, and with copper there is nearly a balance of powers, or changes of power, dependent upon the circumstances detailed in the last section.

When, in electrical combinations containing one metal, water or a neutro-saline solution is in one of the cups, and alkali or acid in another, the result is usually such as might be anticipated,- the side of the metal in the alkali is positive, that in the acid negative, and that in the neutro-saline solution in the opposite state. There are however certain neutrosaline solutions, which when they contain oxygen or common air, act upon the more oxidable metals, and such have a power or energy of their own; thus zinc, and tin, and copper in solution of common salt, are positive to the same metals in distilled water; and the surfaces of the same metals in weak muriatic acid are positive with respect to the surfaces in water or saline solutions. In combinations, in which weak and strong solutions of acids or of alkalies are the two fluids, both being of the same kind, the electrical action is usually feeble; but the surface in the strongest alkali is most positive, and in the acids the result usually depends upon the "nature of the solution; if oxide is formed and deposited, the strongest acid is negative with respect to the diluted one.

The chemical changes produced in combinations of this kind, are best observed in cases where the metals undergo no change; for instance, with platinum, diluted sulphuric acid, and solution of potassa. In this combination, hydrogen soon appears on the platinum in the acid, and a very small quantity 
of gas, which is probably oxygen, on the platinum in contact with the alkali; and that the acid tends to circulate towards the negative surface, and the alkali towards the positive, is shown by the circumstance of the rapid neutralization of the two menstrua, though separated by asbestus moistened in distilled water.

\section{Of combinations consisting of two conductors of the more perfect class, and one fuid.}

The order in which metallic bodies exhibit electricities on contact, as is well known, is intimately connected with their relative oxidability, the most oxidable metal being positive with respect to all those below it. This law extends likewise to the newly discovered bases of the alkalies and earths. Potassium and sodium, as $I$ have found by bringing them in contact with zinc in a concentrated solution of alkali, are apparently as much positive with respect to this body, as zinc is with respect to platinum and gold.

There is not however any inherent and specific property in each metal which gives it the electrical character; it depends upon its peculiar state-on that form of aggregation which fits it for chemical change. Thus, zinc in amalgamation with mercury is positive with respect to pure zinc, and the amalgam of tin is in the same state with regard to tin; and the metals of the fixed alkalies in amalgam give the highest positive energy to a mass of mercury some thousands of times their weight.

In general, the electricities developed by metallic contact are of a stronger kind than those resulting from the contact of metals with fluids, so that they are not capable of being changed by them. For instance: zinc in acid is positive with respect to all other metals below it in degree of oxidability, though they are placed in alkalies or solutions of sulphurets; there are however exceptions; for instance, with regard to tin, which, when in a strong solution of potassa, is positive to zinc, in an acid solution; and with respect to iron, which, though positive with regard to copper in all acid or neutrosaline fluids, is negative to it in solution of sulphurets or of alkalies. The electro-motion in these instances produced by the contact of the fluids prevailing over that produced by the contact of the metals.

And knowing the energies of the acid and alkaline fluids, it is easy to apply them so as to diminish or enhance the electrical effects developed by metallic contact.

If, for instance, in a combination containing zinc and platinum, we use two fluids, and place the acid in contact with 
the zinc, and the alkali with the platimum, the effect will be exceedingly feeble compared with that produced if the order be reversed, and the zinc be in contact with the alkali, and the platinum with the acid.

The chemical changes taking place in combinations of this kind are always such as tend to restore the equilibrium; the hydrogen and the alkaline body always passing to the negative, and oxygen and the acid to the positive metal.

There is no instance of continued electro-motion except in cases where chemical changes can take place, for even De Luc's or Zamboni's columns do not act when quite dry, and the silver in combinations of this kind, when the negative metal is gold, is uniformly found tarnished: for the exhibition of electricities of tension, however, a very slight chemical action is sufficient, as the quantity of electricity required to give repulsion to light bodies is exceedingly small; but to form electro-magnetic combinations the chemical agents must be of an energetic kind.

As most of the fluids which act powerfully in voltaic combinations contain water, or oxygen and hydrogen, it has been suspected that these principles were essential to the effect: this however does not seem to be the case, for I found zinc and platinum formed powerful electro-motive circles in fused litharge and fused oxy-chlorate of potassa, which are not known to contain water; and I have little doubt that similar effects would be produced by other fused salts containing only acid and alkaline matter.

It may elucidate this part of the subject, which must at best be obscure, to take a view of the changes occurring in one of the simplest voltaic combinations,-that consisting of zinc, platinum, and solution of sulphate of soda. It is a fact that zinc and platinum become electrical by contact, the zinc positive, the platinum negative; and the two kinds of electricity are apparently most intense at the surfaces where they are in contact with the fluid, which is too imperfect a conductor to allow them to neutralize or destroy each other: they consequently exert their attractive and repellent powers upon the elements of the menstruum; acid and oxygen circulate to the surface of zinc, which in consequence is dissolved, and alkali and hydrogen to the surface of platinum, of which the hydrogen is disengaged, and the equilibrium broken by the contact of the metals is restored by the chemical changes; so that a constant circulation, or a current of electricity, takes place, the power of the combination becoming feebler in proportion as the solution is decomposed, and acid accumulated round its positive, and alkali round its negative surface. 
In cases where acids or acid solutions alone are used, the destruction of one or both surfaces, with the transfer of hydrogen or oxygen, seems to produce the same effect; and the inactivity of single circles or voltaic piles, in which pure water is used, or saline solutions freed from air, seems to show that the destruction of the surface of the oxidable metal is one of the conditions of continued electrical action; and the cessation of the power of De Luc's or Zamboni's piles, is always connected with the tarnish of the imperfect metal employed in them.

Having published many years ago tables of the electro-chemical relations of metals, which have been copied into many elementary books, I think it proper to give them here in a corrected form with some additions, and the differences dependent upon the nature of the menstruum. The metal mentioned first is positive to all those below it in the scale.

\section{With common acids.}

Potassium and its amalgams; barium and its amalgams; amalgam of zinc; zinc; amalgam of arnmonium (?); cadmium, tin, iron, bismuth, antimony (?), lead, copper, silver, palladium, tellurium, gold, charcoal, platinum, iridium, rhodium.

With alkaline solutions.

The alkaline metals and their amalgams: zinc, tin, lead, copper, iron, silver, palladium, gold, platinum, \&c.

With solutions of hydro-sulphurets.

Zinc, tin, copper, iron, bismuth, silver; platinum, palladium, gold, charcoal.

[To be continued.]

XXIII. A Mode of Heating Water for a Bath. By EDWard Deas Thomson, Esq.*

CONVINCED of the great utility of the warm bath to health, as well as the comfort of it, I have for some time turned my attention to the best and most oconomical mode of heating a bath; and have endeavoured, as far as possible, to obviate the inconvenience, delay and expense, which are inseparable from the greater number of the methods hitherto in use. The result has been to exceed my sanguine hopes; having obtained a bath containing 40 gallons of water at a temperature of $98^{\circ}$ Fahrenheit, in the space of half an hour from the time of lighting the fire. The quantity of coals consumed was under 7 pounds, and the whole expense in London, including the

* Communicated by the Author. 